\title{
Análise das prescrições de sibutramina dispensadas em drogarias no município de Cuiabá-MT, Brasil
}

\author{
Sibutramine prescription at drugstores from Cuiabá - MT, Brazil
}

Recebido em: 08/12/2014 Aceito em: 28/02/2015
Josyanne Barros GONZAGA, Franciele da Silva SIQUEIRA, Jéssica Teixeira ZAMPRONE, Bruna FRANCO, Sthephane Keith Correia CONSTANTINO, Ednaldo Anthony Jesus SILVA, Ruberlei Godinho de OLIVEIRA Faculdade de Farmácia, Universidade de Cuiabá, Avenida Beira Rio, $n^{\circ} 3100$, CEP: 78025-190. Cuiabá-MT, Brasil.E-mail: ruberlei_godinho@hotmail.com

\begin{abstract}
Obesity is a metabolic disorder characterized by body mass increase or an excess of adipose tissue in the body. Treatment of this disease is complex and requires a multidisciplinary attention based on pharmacological, nonpharmacological and/or surgical interventions. Among the pharmacological options, sibutramine, is the most suitable drug for overweight individuals. The objective of this work was to analyze sibutramine prescriptions dispensed in drugstores of Cuiabá, Mato Grosso, Brazil in relation to the distribution of age, gender, medical specialty, as well as to obtain the main prescriptions errors of this medication. Eight hundred and fifteen (815) special control revenue, B2, Sanitary Surveillance in Municipality of Cuiabá-MT, stemming from five random sampling of drugstore chain in the months of July, August and September of 2014. Of which, 83, 7\% corresponded to female users and the rest male. Regarding age, there was a predominance of 31-35 years and $9.94 \%$ was hidden from patient age. The prescription of sibutramine was prevalent among general practitioners, followed by endocrinologists, and being among the items mandatory to be filled in B2 prescription there were no errors in filling out the forms. According to assessment of the prescriptions, it appears that the use of sibutramine is prescribed without accurate diagnosis of the degree of obesity, which causes public health issues, as well as irrational use of this anorectic agent drug by Cuiaba population.
\end{abstract}

Keywords: obesity; notifications; anorectic agent; sibutramine

\section{RESUMO}

A obesidade é uma doença metabólica caracterizada pelo aumento de massa corporal e/ou por um excesso de tecido adiposo no organismo. Dentre as opções farmacológicas, a sibutramina, é o medicamento mais indicado para indivíduos com sobrepeso. O objetivo do trabalho foi analisar as prescrições de sibutramina dispensadas nas drogarias de Cuiabá, Mato Grosso, Brasil em relação à distribuição da faixa etária, sexo, especialidade médica, bem como obter quais os principais erros de prescrições deste medicamento. Foram analisadas no período de três meses 815 receitas de controle especial, B2, na Vigilância Sanitária do município de Cuiabá-MT, decorrentes de cinco drogarias de rede aleatórias nos meses de julho, agosto e setembro do ano de 2014. Das quais, 83,7\%, corresponderam a usuários do sexo feminino e restante ao sexo masculino. Quanto à faixa etária, observou-se a predominância entre 31-35 anos e em $9,94 \%$ foi ocultada a idade do paciente. A prescrição de sibutramina foi prevalente por clínicos gerais, seguido de endocrinologistas, sendo que entre os itens obrigatórios a ser preenchidos no receituário B2 não houve erros no preenchimento. De acordo com avaliação dos receituários, constata-se que o uso de sibutramina é prescrito sem diagnóstico preciso do grau de obesidade, o que acarreta problemas de saúde pública, bem como o uso irracional deste medicamento anorexígeno pela população cuiabana.

Palavras chave: obesidade; prescrição; anorexígeno; sibutramina 


\section{INTRODUÇÃO}

A obesidade é uma doença metabólica caracterizada pelo aumento de massa corpórea ou por um excesso de tecido adiposo no organismo. Os fatores psíquicos, sociais, orgânicos, vida sedentária, má alimentação e a ingestão de alimentos ricos em gorduras são responsáveis pela fisiopatologia desta doença (1). Essa patologia promove morbidade de várias outras doenças, como diabetes, dislipidemia, doenças vasculares, articulares, neoplásicas e hepáticas (2).

O diagnóstico da obesidade, de acordo com dados epidemiológicos, é feito a partir do Índice de Massa Corporal (IMC), obtido pelo quociente entre a divisão do peso (quilogramas) e o quadrado da altura (metros). O excesso de peso é diagnosticado quando o IMC alcança valor igual ou superior a $25 \mathrm{~kg} / \mathrm{m}^{2}$, enquanto que a obesidade é diagnosticada a partir do IMC de $30 \mathrm{~kg} / \mathrm{m}^{2}$ (3).

Segundo dados do Ministério da Saúde, a cidade de Cuiabá, tem 50,4\% da população adulta com excesso de peso, destacando-se em primeiro lugar como a capital mais obesa do Brasil com média de 18,7\% (4).

O tratamento de obesidade é complexo e exige uma atenção multidisciplinar, além de associação de tratamentos e mudanças de estilo de vida. As opções de tratamento para obesidade são intervenções farmacológicas, não farmacológicas e/ou cirúrgicas. Dentre as opções farmacológicas, hoje a sibutramina é o medicamento mais indicado para indivíduos com sobrepeso e morbidades associadas ou quando os tratamentos convencionais falharam (5).

A sibutramina é um fármaco derivado fenetilamínico, inibidor da receptação da serotonina e da norepinefrina, o que induz a diminuição do consumo alimentar e aumenta a termogênese. Em vários ensaios clínicos, a sibutramina promoveu redução estatisticamente significante do peso, da concentração de colesterol total, triglicerídeos, LDL-colesterol e hemoglobina glicada de pacientes obesos diabéticos ou não obesos (6).

Tendo em vista que esse fármaco atua sobre o sistema nervoso central (SNC), é classificado como psicotrópico e deve ser utilizado exclusivamente sob a prescrição e acompanhamento médico. No Brasil, o comércio de medicamentos psicotrópicos é regulado pela Portaria $n^{\circ} 344 / 1998$ da Anvisa, que estabelece que os medicamentos sujeitos a controle especial, contendo, entre outros, fármacos anorexígenos pertencem à Lista B2 e devem ser dispensados mediante apresentação de notificação de receita B (7).

Em farmácias ou drogarias, esta portaria normatiza que a dispensação de sibutramina seja feita quando todos os itens da receita estiverem devidamente preenchidos, juntamente com o termo de responsabilidade do prescritor, segundo a RDC no 52/2011. A responsabilidade pela notificação cabe aos profissionais de saúde, aos detentores do registro de medicamentos contendo a substância sibutramina, seus sais e isômeros bem como intermediários (8).

Este estudo teve como objetivo analisar as prescrições do uso de sibutramina dispensadas nas drogarias de Cuiabá, Mato Grosso, Brasil em relação á sua distribuição, principal faixa etária, sexo, identificar a especialidade médica prescritora, bem como obter quais os principais erros de prescrições deste medicamento.

\section{MÉTODO}

Foi realizado um estudo transversal de caráter quantitativo e descritivo. A amostra foi constituída por todas as prescrições/notificações, contendo sibutramina, dispensadas em cinco drogarias nos meses de julho, agosto e setembro do ano de 2014, escolhidas aleatoriamente, retidas na Vigilância Sanitária Municipal (VISA) do município de Cuiabá, Mato Grosso, Brasil.

Foi elaborado um formulário no qual foram registradas a presença ou ausência dos itens obrigatórios em cada prescrição/notificação, idade e sexo, além da especialidade do prescritor. Entre os itens obrigatórios para as prescrições/notificações listados no Art. $36^{\circ}$ da Portaria $n^{\circ} 344 / 1998$, foram avaliados identificação do emitente, endereço do consultório médico, número da inscrição no Conselho Regional de Medicina, assinatura do prescritor, nome e endereço completos do usuário, nome do medicamento prescrito sob a DCB, dosagem ou concentração, posologia, número de unidades prescritas em algarismos arábicos e por extenso, data da prescrição e forma farmacêutica. Os dados obtidos foram digitados em planilhas do Excel $^{\circledR}$ para a realização dos cálculos, apresentados como média e frequência percentual (\%). A pesquisa atende a resolução $n^{\circ} 466 / 2012$ e foi aprovada pela Plataforma Brasil, sob o número de ata 934.876.

\section{RESULTADOS E DISCUSSÃO}

No período de três meses, foram analisadas 815 receitas de controle especial na VISA do município de Cuiabá-MT, decorrentes de cinco drogarias aleatórias pertencentes a redes diferentes dos meses de julho, agosto e setembro do ano de 2014. A análise dos dados permitiu observar predominância de pacientes do sexo feminino no montante de $684(83,93 \%)$, e os homens apresentando apenas 16\% (131 indivíduos) dessa população de uso. 
Em relação à faixa etária, foi observado que $17,91 \%$ dos pacientes possuíam idade entre 31 e 35 anos. Com base na Tabela 1, em 81 (9,94\%) das prescrições ocorreu erro de preenchimento nas receitas, ocultando a idade do paciente. Segundo a RDC 52/2011 (8) o preenchimento correto da idade é obrigatório pelo prescritor, devido à contraindicação em pacientes com idade acima de 65 (sessenta e cinco) anos, crianças e adolescentes.

Tabela 1: Faixa etária dos pacientes que fazem uso de sibutramina em drogaria em Cuiabá, MT, entre os meses de julho e setembro de 2014

\begin{tabular}{|c|r|r|}
\hline Faixa etária & \multicolumn{1}{|c|}{$\mathbf{n}$} & \multicolumn{1}{c|}{$\%$} \\
\hline Sem idade informada & 81 & 9,94 \\
\hline$<15$ anos & 0 & 0,00 \\
\hline $15-20$ anos & 14 & 1,72 \\
\hline $21-25$ anos & 82 & 10,06 \\
\hline $26-30$ anos & 129 & 15,83 \\
\hline $31-35$ anos & 146 & 17,91 \\
\hline $36-40$ anos & 120 & 14,72 \\
\hline $41-45$ anos & 84 & 10,31 \\
\hline $46-50$ anos & 68 & 8,34 \\
\hline$>50$ anos & 91 & 11,17 \\
\hline Total & 815 & $\mathbf{1 0 0 , 0 0}$ \\
\hline
\end{tabular}

Dentre as justificativas de sobrepeso, pré-cirúrgico e obesidade, a prevalência de uso de sibutramina foi predominante em casos de obesidade graus 1 e 2 (77,18\%, 629 indivíduos), seguido de sobrepeso (161 indivíduos, $19,75 \%$ ), e alguns casos sem justificativa para uso $(3,07 \%, 25)$, sendo considerada uma falha na prescrição.

Com relação ao número de comprimidos prescritos, foi observada alta frequência da apresentação com 30 comprimidos em 800 receituários $(98,16 \%)$, sendo esta a quantidade padronizada pelas indústrias farmacêuticas. De acordo com a RDC 52/2011 (8), a quantidade prescrita de sibutramina é para tratamento máximo de um mês.

Foi observado o predomínio de comprimidos com concentração de $15 \mathrm{mg}$ (661 receitas, 81,10\%). Esta dosagem é referência estabelecida para medicamentos industrializados; em outra vertente foram notadas concentrações de $10 \mathrm{mg}$ de sibutramina $(154,18,90 \%)$.

Analisando os medicamentos dispensados, foi observado que em 682 receitas $(83,68 \%)$ ocorreram os classificados como os de referência, seguidos de 82 $(10,06 \%)$ similares e $47(5,77 \%)$ genéricos. Os medicamentos de referência são mais caros que os outros, po- rém os médicos priorizam o uso destes, justificando a grande quantidade de dispensação em drogarias.

Com relação à especialidade do prescritor, foi constatado que há predominância de clínico geral (421, $51,66 \%)$, seguido de endocrinologista $(230,28,22 \%)$ e em $15 \%$ dos receituários outras especialidades (Tabela 2)

Tabela 2: Especialidade dos prescritores em prescrições e sibutramina em drogarias de Cuiabá, MT, entre os meses de julho e setembro de 2014.

\begin{tabular}{|lrr|}
\multicolumn{1}{r}{ Especialidade } & $\mathrm{n}$ & \% \\
\hline Clínico geral & 421 & 51,66 \\
\hline Endocrinologista & 230 & 28,22 \\
\hline Outros & 129 & 15,83 \\
\hline Psiquiatra & 17 & 2,09 \\
\hline Ginecologista & 11 & 1,35 \\
\hline Neurologista & 0 & 0,00 \\
\hline Hematologista & 0 & 0,00 \\
\hline Pneumologista & 0 & 0,00 \\
\hline Clínico cirúrgico & 7 & 0,00 \\
\hline Cirurgião plástico & 0 & 0,00 \\
\hline \multicolumn{1}{r}{ Total } & 815 & 100 \\
\hline
\end{tabular}

No que se refere aos itens obrigatórios em receitas B2, todas as prescrições analisadas (815) apresentavam os seguintes itens: identificação do paciente, endereço do consultório médico, número de inscrição no Conselho Regional de Medicina (CRM), nome e endereço completos do paciente, nome do fármaco conforme a DCB, dosagem ou concentração, forma farmacêutica, posologia, número de unidades prescritas em números arábicos e por extenso, data da prescrição e assinatura do prescritor.

A RDC n 52/2011, referente à notificação da prescrição e da dispensação de fórmulas ou medicamentos que contenham sibutramina, regulamenta o controle dos itens obrigatórios do receituário B2 (8).

Das prescrições analisadas e seus itens obrigatórios, o uso de sibutramina, foi predominante entre pacientes do sexo feminino. Os resultados encontrados são similares em estudos de Tiyo e cols. (2012), com prevalência de $99 \%$ do sexo feminino e somente $1 \%$ de pacientes do gênero masculino, em estudo realizado entre outubro de 2011 e março de 2012, pelo uso do anorexígeno dispensados em 05 farmácias na cidade de Paranavaí, no noroeste do Paraná (9). Segundo os autores, essa divergência está diretamente relacionada com os padrões de beleza imposta pela sociedade moderna. 
Quando foi analisada a faixa etária dos pacientes, foi observado que a maioria compreendeu a faixa etária de 31-35 anos, sendo que não foi classificado segundo sexo. O mesmo intervalo de idade foi relatado por Cruz (2013) (10). Entretanto, um dado relevante é que em cerca de $9,94 \%$ das prescrições não foram declaradas a idade do paciente, infringindo o art. $2^{\circ}$ da RDC n ${ }^{\circ} 52 / 2011$, que trata do termo de responsabilidade: a idade, deve ser preenchida corretamente pelo prescritor e a prescrição deve ser devidamente assinada pelo paciente (8).

$\mathrm{Na}$ avaliação da justificativa de uso, entre elas sobrepeso, tratamento pré-cirúrgico e obesidade, a predominância de uso em casos de obesidade foi de $77,18 \%$ dos usuários, sendo classificada em obesidade de grau 1 e 2, seguido de sobrepeso, Em alguns casos a prescrição estava sem justificativa para uso, sendo considerada uma falha. Segundo Souza (2012) o uso de fármacos moderadores de apetite em obesos é considerado como medida coadjuvante das modificações de estilo de vida (11).

De acordo com a legislação vigente (8), a dosagem máxima de sibutramina é de $15 \mathrm{mg} / \mathrm{dia}$, o que foi encontrado na a maioria das receitas analisadas. Em 18,90\% das prescrições, a dosagem foi $10 \mathrm{mg}$, enquanto a concentração da dosagem predominante foi $15 \mathrm{mg}$ do anorexígeno. Vieiro e cols. (2011) também relataram as mesmas dosagens, que estão de acordo com a legislação vigente. Em relação à quantidade de comprimidos prescritos, foi observado o predomínio de 30 comprimidos, sendo esta a quantidade padronizada pelas indústrias farmacêuticas e compatível com o tempo máximo do tratamento ( 30 dias), estabelecido pela RDC 52/2011 $(8,12)$. Apenas $3(0,37 \%)$ prescrições com 60 comprimidos ou mais foram aviadas neste período, mostrando erro de prescrição médica.

Dos receituários analisados, a maioria $(84 \%)$ foi referente a medicamento de referência seguido de similares e genéricos. Conforme a Lei 9.787/99 é permitida a intercambialidade entre medicamentos de referência pelos genéricos. Entretanto, há uma preferência dos medicamentos de referência tanto pelos profissionais prescritores quanto pela população, devido ao estigma social imposto e preconceito com medicamentos que podem ser intercambiáveis (13). Segundo dados da Associação Brasileira das Indústrias de Medicamentos Genéricos, as apresentações de medicamentos genéricos disponíveis no mercado brasileiro atendem a mais de $60 \%$ das necessidades de prescrições médicas. Segundo Blatt e cols. (2012), o desconhecimento por parte da população, ou mesmo por ainda existirem certos preconceitos, são fatores que inviabilizam a utilização dos medicamentos genéricos (13).

Em relação à especialidade médica, o estudo mostrou que mais da metade das prescrições foram elaboradas por clínicos gerais, seguidos de endocrinologistas. Resultados de Oliveira e cols. (2011) sugeriram que a prescrição/notificação de medicamentos para casos específicos de tratamento de redução de peso, deve ser emitida por médicos endocrinologistas ou especialistas (14). O mesmo pode ser constatado em Santa Catarina, onde $43 \%$ das prescrições foram elaboradas por clínicos gerais, seguidas por endocrinologistas (12). Porém, noo estudo realizado em Cascavel, Paraná, foi observado que $60 \%$ das receitas foram emitidas por endocrinologistas e apenas $10 \%$ por clínico geral (15). Foi sugerido que o número elevado de prescrições por clínicos gerais se dá pela facilidade de acesso a essa especialidade no Sistema Único de Saúde, em contrapartida aos endocrinologistas (16).

Os itens obrigatórios foram devidamente preenchidos, em acordo com a determinação regulamentada pela RDC 344/1998 e RDC 52/2011. Abrantes e cols. (2007) relataram que não basta o diagnóstico preciso e a seleção adequada do medicamento, se a prescrição não for completa em todos seus aspectos e rigorosamente elaborada. Além de ser devidamente preenchida para evitar erros primários, bem como contribuir para o uso racional da sibutramina (17).

\section{CONCLUSÃO}

De acordo com avaliação das receitas, o uso de sibutramina foi prescrito sem diagnóstico preciso do grau de obesidade, o que acarreta problemas de saúde pública, bem como o uso irracional deste anorexígeno pela população. Neste contexto, destaca-se a necessidade da adoção de medidas educativas para prescritores, frente aos aspectos éticos que norteiam a prescrição e dispensação de sibutramina, a fim de evitar o uso indiscriminado a partir de um diagnostico e prescrição equivocada.

\section{AGRADECIMENTOS}

Os autores agradecem à Vigilância Sanitária Municipal de Cuiabá-MT pelo provimento dos dados, bem como os seus colaboradores que contribuíram igualmente para a realização deste estudo e ao Comitê de Ética em Pesquisa de Humanos da Universidade de Cuiabá (UNIC) pela aprovação do projeto. 


\section{REFERÊNCIAS}

1. Brito AF, Vieira AS, Rabelo MM. Avaliação do índice de venda da sibutramina na cidade de Ceres-GO. Rev Refacer. 2013; 2(1):01-9.

2. Mancini MC, Halpern A. Tratamento Farmacológico da Obesidade. Arq Bras Endocrinol Metab. 2002; 5(46):497512 .

3. World Health Organization. Obesity: preventing and managing the globalepidemic. Report of a WHO consultation. World Health Organ Tech Rep Ser. 2000; 894(12):1-253.

4. BRASIL. Ministério da Saúde. Secretaria de Vigilância em Saúde. Secretaria de Gestão Estratégica e Participativa. Vigitel Brasil 2010: vigilância de fatores de risco e proteção para doenças crônicas por inquérito telefônico/ Ministério da Saúde, Secretaria de Vigilância em Saúde, Secretaria de Gestão Estratégica e Participativa. Brasília: Ministério da Saúde, 2011:47-54.

5. Soares VCG, Vechiato C, Pierini EC, Demarchi GM, Francesconi EPMS, Oliveira DAG. Autoimagem corporal associada ao uso de sibutramina. J Health Sci Inst. 2011; 29(1):45-51.

6. Fortes RC, Guimarães NG, Haack A, Torres AAL, Carvalho KMB. Orlistat e sibutramina: bons coadjuvantes para perda e manutenção de peso? Rev Bras Nutr Clin. 2006; 21(3):244-51.

7. BRASIL. Agência Nacional de Vigilância Sanitária. Portaria $n^{\circ} 344$, de 12 de maio de 1998. Aprova o Regulamento Técnico sobre substâncias e medicamentos sujeitos a controle especial. Diário Oficial da União 31 de dez 1998.

8. BRASIL. Agência Nacional de Vigilância Sanitária. Resolução $n^{\circ} 52$, de 6 de outubro de 2011. Dispõe sobre a proibição do uso das substâncias anfepramona, femproporex e mazindol, seus sais e isômeros, bem como intermediários e medidas de controle da prescrição e dispensação de medicamentos que contenham a substância sibutramina, seus sais e isômeros, bem como intermediários e dá outras providências. Diário Oficial da União 13 de out 2011.

9. Tiyo R, Rampi DMV. Prevalência de medicamento contendo cloridrato de sibutramina em farmácias da cidade de Paranavaí-Pr. Uningá Review. 2012; 10(1):104-112.
10. Cruz ACS, Santos EN. Avaliação do consumo de medicamentos para emagrecer em farmácias, no município de Ceres - Goiás, Brasil. Rev Univ Vale do Rio Verd. 2013; 1(10):402-9.

11. Souza FJZN, Rau C. O uso da sibutramina em pacientes obesos e seu efeito sobre a pressão arterial [apresentação na $7^{\circ}$ Mostra de Produção Científica da Pós-Graduação Lato Sensu da PUC Góias, 2012. Out 1633-1647; Goiás, Brasil].

12. Locateli C, Viero TR. Perfil de prescrição e comparativo de vendas de sibutramina antes e após RDC n ${ }^{\circ} 25$ de 30 de junho de 2010 e RDC N 52 de 06 de outubro de 2011 em uma farmácia da cidade de Vieira - SC. RIES. 2013; (2)1: 8-17.

13. Blatt CR, Trauthman SC, Schmidt EH, Marchesan S, Silva LM, Martins JL. Conhecimento popular e utilização dos medicamentos genéricos na população do município de Tubarão, SC. Ciênc. saúde coletiva. 2012; 17(1):79-87.

14. BRASIL. Ministério da Saúde. Agência Nacional da Vigilância Sanitária. Lei n. 9787 de 10 de fevereiro de 1999. Altera a Lei $\mathrm{n}^{\circ}$ 6.360, de 23 de setembro de 1976. Dispõe sobre a vigilância sanitária, estabelece o medicamento genérico, dispõe sobre a utilização de nomes genéricos em produtos farmacêuticos e dá outras providências. Diário Oficial da União, poder executivo 11 de fevereiro 1999a.

15. Oliveira KR, Buzanelo VV. Análise das prescrições de medicamentos usados no tratamento da obesidade dispensadas em drogaria no município de Ijuí- RS. Rev Ciênc Farm Básica Apl. 2011; 32(3):381-7.

16. Henning K, Wiens A, Sanches ACC. Estudo das prescrições de anorexígenos dispensados em uma farmácia com manipulação de cascavel-pr. Visão Acadêmica. 2011; 12(2):29-40.

17. Borges GPM, Belo T, Vieira MM, Vieira JRS. Análise dos receituários de medicamentos psicotrópicos anorexígenos em uma rede privada de farmácia de Belém-pará. Infarma. 2008; 9/10(10):26-30

18. Abrantes PM, Magalhães SMS, Acúrcio FA, Sakurai E. Avaliação da qualidade das prescrições de antimicrobianos dispensadas em unidades públicas de saúde de Belo Horizonte, Minas Gerais, Brasil, 2002. Cad Saúde Pública. 2007; 23(1):95-104 